
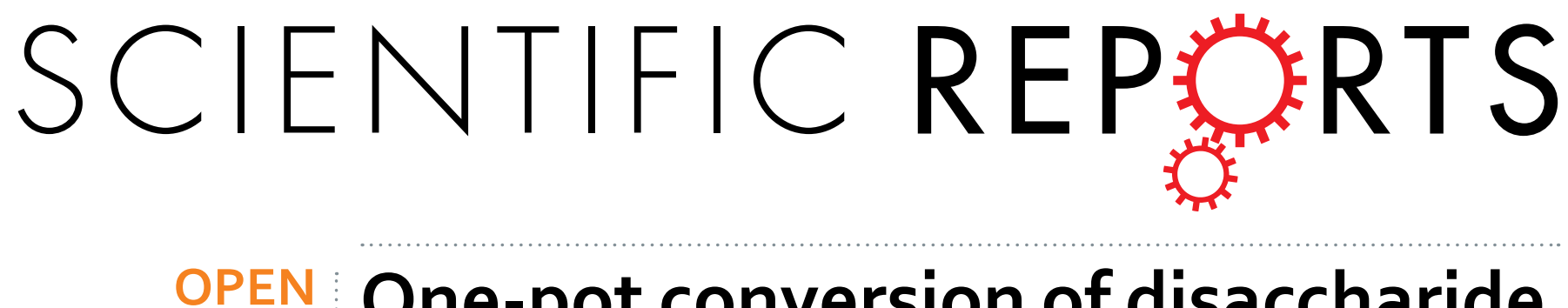

\title{
One-pot conversion of disaccharide into 5-hydroxymethylfurfural catalyzed by imidazole ionic liquid
}

Received: 25 January 2016

Accepted: 26 April 2016

Published: 16 May 2016
Yongshui $\mathrm{Qu}^{1}$, $\mathrm{Li} \mathrm{Li}^{1,2}$, Quanyuan Wei ${ }^{3}$, Chongpin Huang ${ }^{2}$, Piotr Oleskowicz-Popiel ${ }^{4}$ \& Jian Xu ${ }^{1}$

Conversion of carbohydrate into 5-hydroxymethylfurfural (5- HMF), a versatile, key renewable platform compound is regarded as an important transformation in biomass-derived carbohydrate chemistry. A variety of ILs, not only acidic but also alkaline ILs, were synthesized and used as catalyst in the production of 5-HMF from disaccharide. Several factors including reaction temperature, IL dosage, solvent and reaction time, were found to influence the yield of 5-HMF from cellobiose. Of the ILs tested, hydroxy-functionalized ionic liquid (IL), 1-hydroxyethyl-3-methylimidazolium tetrafluoroborate ([AEMIM]BF ${ }_{4}$ ) showed the highest catalytic activity and selectivity. 5 -HMF yield of $68.71 \%$ from sucrose was obtained after $6 \mathrm{hrs}$ at $160^{\circ} \mathrm{C}$. At the same condition with cellobiose as substrate, 5 -HMF yield was $24.73 \%$. In addition, 5 -HMF also exhibited good stablity in this reaction system. Moreover, a kinetic analysis was carried out in both acidic and alkaline IL-catalyzed system, suggesting main side reaction in the conversion of fructose catalyzed by acidic and alkaline IL was polymerization of fructose and 5-HMF degradation, respectively.

The massive consumption of fossil resources has made it an essential task to find new feedstocks. Biomass has a remarkable potential to serve as an alternative of fossil resources for the production of energy and organic compounds ${ }^{1-3}$. 5-hydroxymethylfurfural (5- HMF) has been reported to be one of the top building block chemicals obtained from biomass ${ }^{4,5}$. A broad range of value added compounds can be synthesized from it, such as 1,6-hexanediol ${ }^{6}$, 5-hydroxymethyl-2-furancarboxylic acid (HMFCA) ${ }^{7}$, 5-ethoxymethylfurfural (EMF) ${ }^{8}$, 2,5-dihydroxymethylfurfural (DHMF) and 5-hydroxymethyl furanoic acid (HMFA) ${ }^{9}, 2,5$-furandicarboxylic acid $^{10}, 2,5$-diformylfuran $(2,5 \text {-DFF })^{11}, 5$-formyl-2-furancarboxylic acid (FFCA) and 2,5-furandicarboxylic acid $(\text { FDCA })^{12}$.

Since the emergence of room temperature ionic liquids (ILs), it has been playing an important role in various aspects of chemical researches. The distinctive properties give ILs a chance to be used extensively in catalysis ${ }^{13,14}$ and subsequently in biomass processing ${ }^{15,16}$. In marked contrast to many studies with the application of ILs as solvent or co-solvent on 5-HMF production ${ }^{5,17}$, very few have been focused on ILs' catalytic activity. Although there is a growing literature on dehydration of C-6 carbohydrates, mainly fructose and glucose ${ }^{18,19}$, for the production of 5-HMF, little is available on conversion of dissacharide. When 1-methyl-3-octylimidazolium chloride was used as solvent and $\mathrm{CrCl}_{2} / \mathrm{HCl}$ as catalyst, 5-HMF yield could reach $82 \pm 3.7 \%$ from sucrose, higher than that with $\mathrm{ZnCl}_{2} / \mathrm{HCl}$ as catalyst which was $58 \pm 2.7 \%{ }^{20}$. Ilgen ${ }^{21}$ demonstrated the production of 5-HMF in ILs with the presence of $\mathrm{CrCl}_{2}$ and a maximum 5-HMF yield of $42 \%$ was obtained from the dehydration of sucrose under $100^{\circ} \mathrm{C}$ for $1 \mathrm{~h}$. Lima et al..$^{22}$ reported a 5 - HMF yield of $58 \%$ achieved using [BMIM] Cl as reaction medium with a mass ratio of IL to sucrose at 10 with $\mathrm{CrCl}_{3} \cdot \mathrm{H}_{2} \mathrm{O}$ at $100^{\circ} \mathrm{C}$ for $4 \mathrm{~h}$. The conversion of sucrose and cellobiose to 5-HMF catalyzed by Lewis acid $\left(\mathrm{SnCl}_{4}\right)$ was studied by $\mathrm{Hu}$ et al. ${ }^{23}$, using 1-ethyl-3-methylimidazolium tetrafluoroborate $\left([\mathrm{EMIM}] \mathrm{BF}_{4}\right)$ as solvent at $100^{\circ} \mathrm{C}$ for $3 \mathrm{~h}$ and obtained the highest yield of $57 \%$ and $65 \%$, respectively. All the above-mentioned ILs are neutral or acidic, and dehydration is promoted due to enhanced dissolution of catalysts and substrates. Although alkaline ILs, display high activity for some Brønsted base-catalyzed reactions (Knoevenagal condensation reaction and Michael addition ${ }^{24,25}$ ) and also can be switched repeatedly by

${ }^{1}$ State Key Laboratory of Biochemical Engineering, Institute of Process Engineering, Chinese Academy of Sciences, Beijing, 100190, China. ${ }^{2}$ Beijing University of Chemical Technology, Beijing, 100029, China. ${ }^{3}$ Beijing Municipal Research Academy of Environmental Protection, Beijing, 100037, China. ${ }^{4}$ Institute of Environmental Engineering, Faculty of Civil and Environmental Engineering, Poznan University of Technology, Berdychowo 4, 60-965, Poznan, Poland. Correspondence and requests for materials should be addressed to J.X. (email: super_xujian@yahoo.com orjxu@ipe.ac.cn) 


\begin{tabular}{|l|l|l|c|c|}
\hline Entry & ILs & Solvent & Time (min) & 5-HMF yield (\%) \\
\hline 1 & {$[\mathrm{MIMPS}]_{3} \mathrm{PW}_{12} \mathrm{O}_{40}$} & 2-Butanol & 180 & $30.29 \pm 1.14$ \\
\hline 2 & {$[\mathrm{MIMPS}]_{3} \mathrm{PW}_{12} \mathrm{O}_{40}$} & DMAc & 300 & $25.92 \pm 0.78$ \\
\hline 3 & {$[\mathrm{MIMPS}]_{3} \mathrm{PW}_{12} \mathrm{O}_{40}$} & NMP & 150 & $15.81 \pm 0.48$ \\
\hline 4 & {$[\mathrm{MIMPS}]_{3} \mathrm{PW}_{12} \mathrm{O}_{40}$} & DMSO & 360 & $18.90 \pm 0.78$ \\
\hline 5 & {$[\mathrm{BMIM}] \mathrm{HCO}_{3}$} & DMSO & 180 & $41.17 \pm 1.44$ \\
\hline 6 & {$[\mathrm{EMIM}] \mathrm{OH}$} & DMSO & 30 & $46.29 \pm 1.22$ \\
\hline 7 & {$[\mathrm{BMIM}] \mathrm{OH}$} & DMSO & 180 & $54.39 \pm 1.19$ \\
\hline 8 & {$[\mathrm{AEMIM}] \mathrm{BF}_{4}$} & DMSO & 480 & $68.71 \pm 0.90$ \\
\hline 9 & {$[\mathrm{AEMIM}] \mathrm{BF}_{4}$} & DMSO & 30 & $35.46 \pm 1.38$ \\
\hline 10 & {$[\mathrm{AEMIM}] \mathrm{BF}_{4}$} & DMSO & 180 & $62.16 \pm 1.41$ \\
\hline
\end{tabular}

Table 1. Dehydration of sucrose with different ILs. Conditions: $0.5 \mathrm{~g}$ substrate, $0.25 \mathrm{~g} \mathrm{ILs,} 60 \mathrm{~mL}$ solvent, $160^{\circ} \mathrm{C}$.

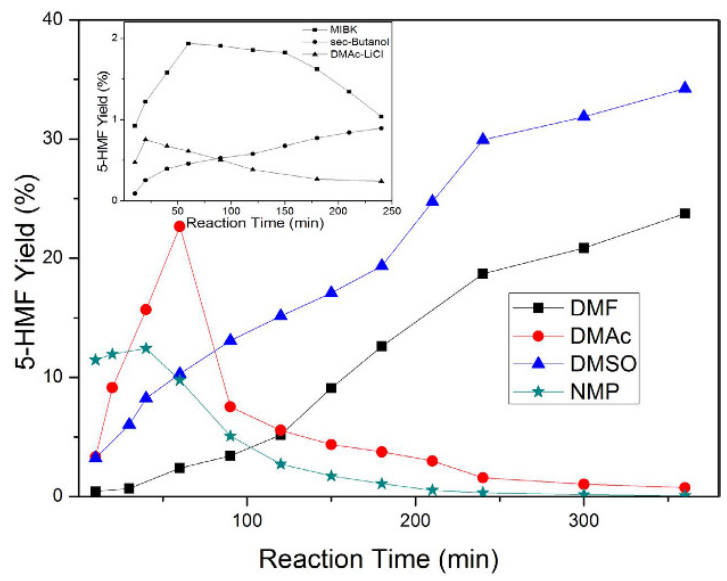

Figure 1. Effect of solvents on 5-HMF yield. Conditions: $0.25 \mathrm{~g}$ [AEMIM]BF, $0.5 \mathrm{~g}$ cellobiose, $60 \mathrm{~mL}$ solvent, at $160^{\circ} \mathrm{C}$, with the presence of $\mathrm{N}_{2}$.

bubbling $\mathrm{CO}_{\mathrm{X}}$ and $\mathrm{NO}_{\mathrm{X}}$ through the solution alternately ${ }^{26-29}$, to our knowledge there have been little reports of the use of alkaline ILs as a catalyst for the conversion of carbohydrates to 5-HMF.

In the present study, Brønsted acidic IL, 1-(3-sulfonic acid) propyl-3-methylimidazolium phosphotungstate ([MIMPS $]_{3} \mathrm{PW}_{12} \mathrm{O}_{40}$ ), and a variety of alkaline ILs, 1-butyl-3-methylimidazolium hydroxide ([BMIM]OH), 1-ethyl-3-methylimidazolium hydroxide ([EMIM]OH), 1-butyl-3-methylimidazolium carbonate ([BMIM] $\mathrm{HCO}_{3}$ ) and hydroxy-functionalized IL, 1-hydroxyethyl-3-methylimidazolium tetrafluoroborate ([AEMIM]BF 4 ) were synthesized and used as catalyst in the conversion of disacharide (sucrose and cellobiose) to 5-HMF, which has not been reported. In addition, kinetic analysis on reaction catalyzed by different ILs was carried out to identify the catalytic effect of different ILs-catalyst on the conversion of dissacharide to 5-HMF.

\section{Results}

Sucrose conversion with different IL catalysts. The effect of ILs with different cations and anions, employed as catalysts for the transformation of sucrose was shown in Table $1(\mathrm{P}<0.05)$. Among different kinds of solvents used for the conversion of sucrose with [MIMPS] ${ }_{3} \mathrm{PW}_{12} \mathrm{O}_{40}$ as catalyst $(\mathrm{P}<0.05)$ (Table 1 , entry $\left.1-4\right)$, a comparable high yield of 5-HMF was obtained using 2-Butanol as solvent (Table 1, entry 1), compared to DMAc, NMP and DMSO (Table 1, entry 2-4), consistent with the results in a previous study ${ }^{30}$. The 5-HMF yield, catalyzed by alkaline ILs (Table 1, entry 5-8) was invariably higher than that obtained with the acidic catalyst (Table 1, entry $1-4$ ). The highest yield was found to be $68.71 \%$ catalyzed by [AEMIM] BF 4 in DMSO (Table 1 , entry 8 ), suggesting that weak alkaline condition favored the reaction. Moreover, the yield sustained growth during the reaction time (Table 1, entry $8-10$ ) demonstrating that 5-HMF exhibited good stability in this reaction system $(\mathrm{P}<0.05)$.

Effect of composition of the reaction medium on cellobiose conversion. As shown in Fig. 1, there was a huge difference in the conversion of cellobiose into $5-\mathrm{HMF}$ with different solvents $(\mathrm{P}<0.01)$. The highest yield of 5- $\mathrm{HMF}$ in the reaction carried out at $160^{\circ} \mathrm{C}$ in DMSO reached $34.25 \%$ in $6 \mathrm{hrs}$. However, the yield was only $23.77 \%$ under the same condition with DMF as solvent.

For the reaction with DMAc and NMP as solvent, the highest yield of 5-HMF (26.29\% and 12.44\%, respectively) was obtained in 60 mins and 40 mins, followed by a quick reduction. In another 3 solvents, MIBK, 2-butanol and DMAc-LiCl, trace amounts of 5-HMF can be ignored. It might be concluded that 5-HMF sta- 


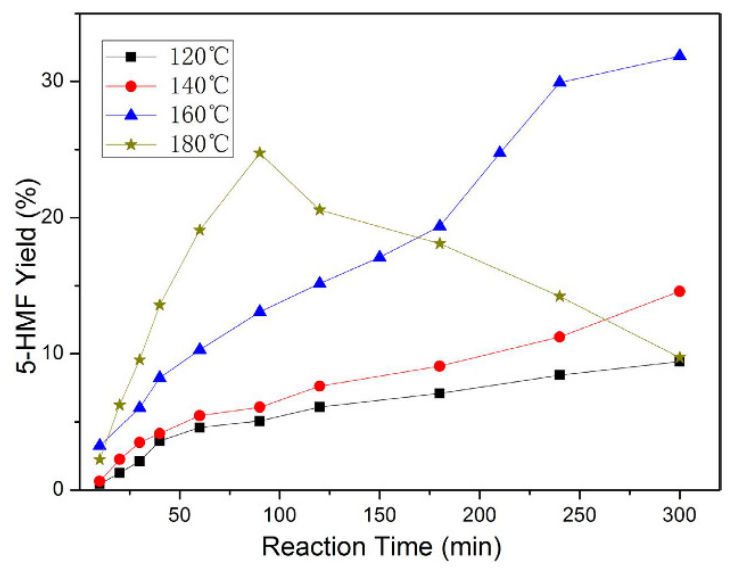

Figure 2. Effect of temperature and time on 5-HMF yield. Conditions: [AEMIM]BF $40.25 \mathrm{~g}$, cellobiose $0.5 \mathrm{~g}$, in $60 \mathrm{~mL}$ DMSO, under $\mathrm{N}_{2}$.

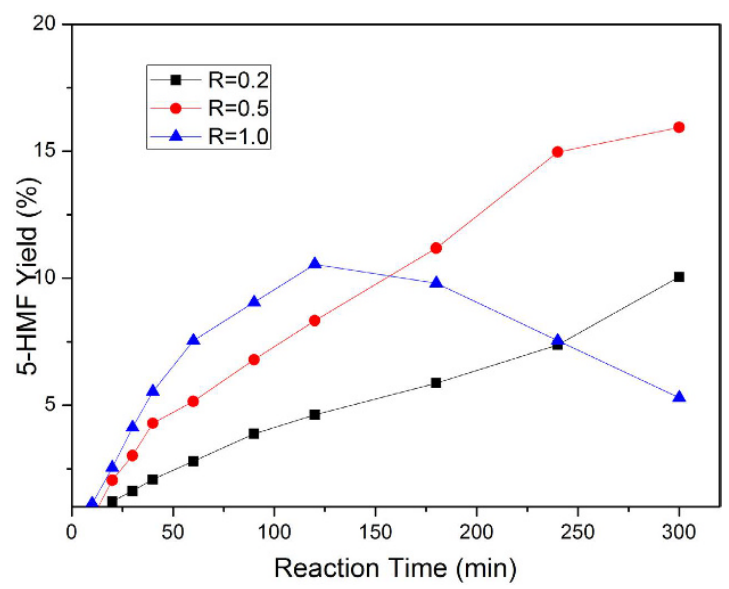

Figure 3. Effect of catalyst amount on 5-HMF yield. Conditions: cellobiose $0.5 \mathrm{~g}, 60 \mathrm{~mL} \mathrm{DMSO}, 160^{\circ} \mathrm{C}$, under $\mathrm{N}_{2}$.

bility is dependent on reaction solvent, i.e., $\mathrm{DMSO}>\mathrm{DMF}>\mathrm{DMAc}>\mathrm{NMP}>\mathrm{MIBK}>2$-butanol $>$ DMAc-LiCl. Moreover, the huge difference between DMAc- $\mathrm{LiCl}$ and DMAc indicates that the addition of metal chloride was not conducive to 5-HMF production, which was different from the reported system with ILs as solvent catalyzed by metal chloride 31,32 .

Effect of temperature and reaction time on cellobiose conversion. Figure 2 shows the effect of temperature and reaction time on the conversion of cellobiose catalyzed by [AEMIM] $\mathrm{BF}_{4}$, using DMSO as solvent $(\mathrm{P}<0.05)$. At $120^{\circ} \mathrm{C}$ and $140^{\circ} \mathrm{C}$, the yields of 5 - HMF increased slowly to $9.44 \%$ and $14.86 \%$ respectively after 300 mins. It presented the similar trend at $160^{\circ} \mathrm{C}$. However, the 5 -HMF formation was obviously enhanced. At $180^{\circ} \mathrm{C}$, although the 5-HMF yield increased rapidly at the initial stage and the maximum 5-HMF yield of $24.73 \%$ was obtained at $90 \mathrm{~min}$, it then dropped dramatically. It could thus be concluded that higher temperature could accelerate the conversion of cellobiose into 5-HMF and in the mean time, it raised the risk of 5-HMF degradation.

Effect of the dosage of IL catalyst on the yield of 5-HMF. Figure 3 shows the effect of the amounts of [AEMIM] $\mathrm{BF}_{4}$ on 5 -HMF yield in the cellobiose transformation reaction $(\mathrm{P}<0.05)$. An evident conclusion could be drawn from Fig. 3 that increasing the ratio of catalyst to cellobiose (R) from 0.2 to 0.5 will promote significantly the conversion of cellobiose to 5 -HMF from $31.87 \%$ to $40.19 \%$ after $5 \mathrm{~h}$, respectively. Further increasing the dosage of catalysts to 1.0, although the time to obtain the maximum 5-HMF yield was reduced to within $2 \mathrm{hrs}$, it was only $21.1 \%$, much lower than that from $\mathrm{R}$ at 0.2 and 0.5 . Moreover, the 5-HMF yield showed a sharp decrease after $2 \mathrm{hrs}$ for $\mathrm{R}$ at 1.0. The reactivity difference might be ascribed to be the fact that IL catalyst typically exhibit high activity not only in conversion of cellobiose, but also in the polymerization of cellobiose, glucose, 5-HMF, and other small molecule compounds during degradation process.

Kinetic analysis of the dehydration of fructose. During conversion of disaccharide into 5-HMF, three reactions are involved, namely the hydrolysis of the disaccharide into its components (fructose and glucose), isomerization of glucose into fructose, and dehydration of fructose. In order to study the process of 5-HMF formation, the kinetic analysis of the dehydration of fructose catalyzed by ILs in DMSO was performed based on two 


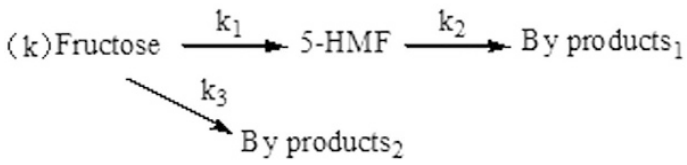

Figure 4. Kinetic model for dehydration of fructose catalyzed by ILs.

\begin{tabular}{|l|c|c|c|c|c|c|}
\hline & Entry & $\mathbf{T}\left({ }^{\circ} \mathbf{C}\right)$ & $\boldsymbol{k}\left(\mathbf{m i n}^{-\mathbf{1}}\right)$ & $\boldsymbol{k}_{\mathbf{I}}\left(\mathbf{m i n}^{-\mathbf{1}}\right)$ & $\boldsymbol{k}_{\mathbf{2}}\left(\mathbf{m i n}^{-1}\right)$ & $\boldsymbol{k}_{\mathbf{3}}\left(\mathbf{m i n}^{-1}\right)$ \\
\hline & 1 & 100 & $0.00399 \pm 2.83 \times 10^{-4}$ & $0.00053 \pm 2.83 \times 10^{-5}$ & $1.3 \mathrm{E}-05 \pm 2.19 \times 10^{-6}$ & $0.003275 \pm 4.74 \times 10^{-4}$ \\
\cline { 2 - 7 } & 2 & 120 & $0.01205 \pm 6.72 \times 10^{-4}$ & $0.009665 \pm 1.34 \times 10^{-4}$ & $0.000116 \pm 7.78 \times 10^{-6}$ & $0.00265 \pm 1.56 \times 10^{-4}$ \\
\cline { 2 - 7 } & 3 & 140 & $0.03202 \pm 0.002638$ & $0.02178 \pm 3.96 \times 10^{-4}$ & $0.000804 \pm 6.54 \times 10^{-5}$ & $0.01121 \pm 8.63 \times 10^{-4}$ \\
\cline { 2 - 7 } & 4 & 160 & $0.08802 \pm 6.20 \times 10^{-3}$ & $0.04375 \pm 7.21 \times 10^{-4}$ & $0.00515 \pm 4.24 \times 10^{-4}$ & $0.037116 \pm 3.2 \times 10^{-3}$ \\
\hline \multirow{4}{*}{ B: Acidic ILs } & 5 & 120 & $0.000262 \pm 7.42 \times 10^{-5}$ & $0.000123 \pm 4.96 \times 10^{-6}$ & $8.89 \mathrm{E}-06 \pm 1.91 \times 10^{-7}$ & $0.000083 \pm 8.49 \times 10^{-7}$ \\
\cline { 2 - 7 } & 6 & 140 & $0.001329 \pm 1.2 \times 10^{-5}$ & $0.000892 \pm 6.15 \times 10^{-5}$ & $2.36 \mathrm{E}-05 \pm 3.04 \times 10^{-6}$ & $0.000408 \pm 1.17 \times 10^{-4}$ \\
\cline { 2 - 7 } & 7 & 160 & $0.008017 \pm 1.46 \times 10^{-4}$ & $0.00596 \pm 1.7 \times 10^{-4}$ & $0.000097 \pm 3.25 \times 10^{-6}$ & $0.001757 \pm 1.08 \times 10^{-4}$ \\
\cline { 2 - 7 } & 8 & 180 & $0.03333 \pm 1.32 \times 10^{-4}$ & $0.02489 \pm 4.53 \times 10^{-4}$ & $0.000341 \pm 3.82 \times 10^{-5}$ & $0.00865 \pm 5.79 \times 10^{-4}$ \\
\hline
\end{tabular}

Table 2. Reaction rate constants $(\mathbf{k})$ of fructose dehydration at different temperatures. Conditions: $0.25 \mathrm{~g}$ catalyst, $0.5 \mathrm{~g}$ fructose, $60 \mathrm{~mL}$ DMSO; acidic and alkaline ILs were [MIMPS] ${ }_{3} \mathrm{PW}_{12} \mathrm{O}_{40}$ and [AEMIM] BF 4 .

step continuous reaction ${ }^{33}$ as shown in Fig. 4: hydrolysis of fructose into 5-HMF and by-products ${ }_{2}$; degradation of 5-HMF into by-products ${ }_{1}$ including formic acid, levulinic acid, humin, and so on. These components, coming from decomposition and polymerization of 5-HMF and fructose, can be analyzed by HPLC or observation, corresponded to some publications ${ }^{34,35}$.

The conversion of 5-HMF is considered as a first-order reaction. 5-HMF generation rate and fructose consumption rate are shown as follows:

$$
\begin{gathered}
\frac{d C_{\text {Fructose }}}{d t}=-k C_{\text {Fructose }}=-k_{1} C_{\text {Fructose }}-k_{3} C_{\text {Fructose }} \\
\frac{d C_{5-H M F}}{d t}=k_{1} C_{\text {Fructose }}-k_{2} C_{5-H M F}
\end{gathered}
$$

Eq. (3) and (4) can be achieved by integrating Eq. (1) and (2), $k_{1}$ and $k_{2}$ are step rate constants, which are constant at a definite temperature:

$$
\begin{gathered}
C_{\text {Fructose }}=C_{\text {Fructose } 0} e^{-k t} \\
C_{5-H M F}=\frac{k_{1} C_{\text {Fructose } 0}}{k_{2}-k_{1}}\left(e^{-k_{1} t}-e^{-k_{2} t}\right)
\end{gathered}
$$

Substituting $C_{\text {Fructose } 0}$ from Eq. (3) into Eq. (4) gives Eq. (5):

$$
Y_{5-H M F}=\frac{k_{1}}{k_{2}-k_{1}}\left(e^{-k_{1} t}-e^{-k_{2} t}\right) \times 100 \%
$$

By integrating the above equation leads to the following:

$$
\begin{aligned}
& \frac{C_{\text {Fructose }}}{C_{\text {Fructose } 0}}=1-X_{\text {Fructose }} \\
& -\ln \left(1-X_{\text {Fructose }}\right)=k t
\end{aligned}
$$

Values of $\ln \left(1-X_{\text {Fructose }}\right)$ (where $X$ is conversion of fructose) are plotted against reaction time $(t)$ at different temperatures in order to obtain rate constants $(k)$. Table 1 shows the relationship between the rate constant and reaction temperature. The results show that $k$ value increases as temperature increases, meaning that the higher temperature accelerates the fructose dehydration reaction rate.

Compared to alkaline system (Table 2 , entry 5-8), $k_{3}$ in acidic system (Table 2 , entry $1-4$ ) grows faster when temperature is increased from $100^{\circ} \mathrm{C}$ to $160^{\circ} \mathrm{C}$. It is why the rate of humin, coming from polymerization of fructose and 5-HMF, lactic acid, etc., rise faster at a higher temperature. 

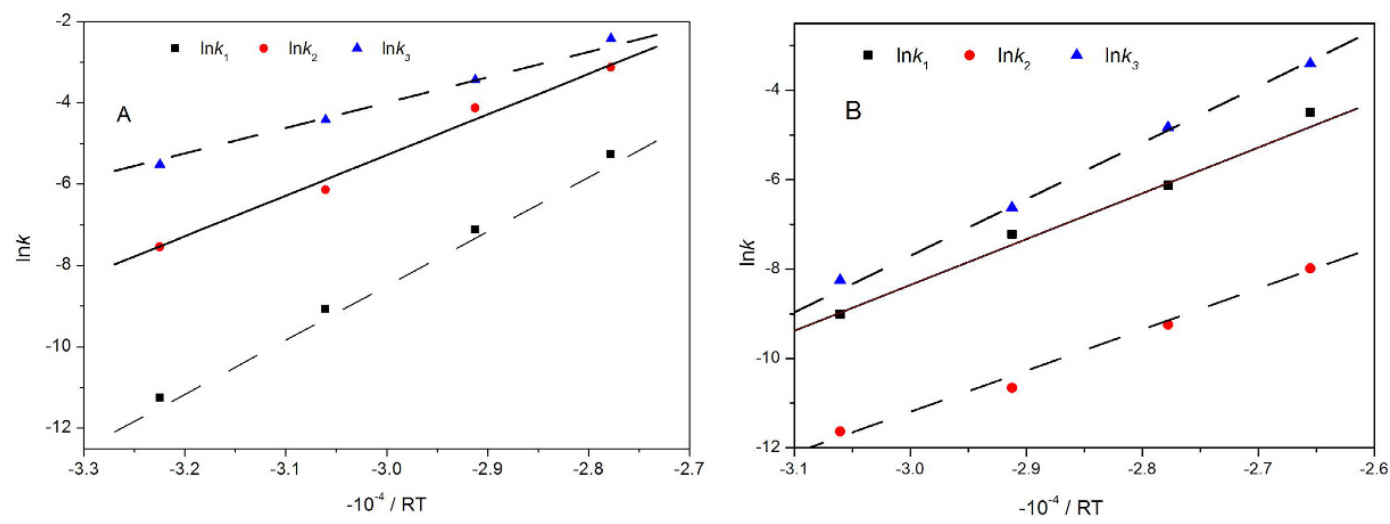

Figure 5. Relationship of $\ln k$ and $-10^{-4} / \mathrm{RT}$.

\begin{tabular}{|l|c|c|c|c|c|c|}
\hline \multirow{2}{*}{} & \multicolumn{3}{|c|}{ Pre-exponential factor, $\mathbf{A}\left(\mathbf{m i n}^{-1}\right)$} & \multicolumn{3}{c|}{ Activation energy, Ea $\mathbf{( k J \cdot \mathbf { m o l } ^ { - 1 } )}$} \\
\cline { 2 - 7 } & $\boldsymbol{A}_{\mathbf{1}}$ & $\boldsymbol{A}_{\mathbf{2}}$ & $\boldsymbol{A}_{\mathbf{3}}$ & $\boldsymbol{E \boldsymbol { a } _ { \mathbf { 1 } }}$ & $\boldsymbol{E} \boldsymbol{a}_{\mathbf{2}}$ & $\boldsymbol{E a}_{\mathbf{3}}$ \\
\hline Acidic ILs & $5.06 \times 10^{10}$ & $4.95 \times 10^{13}$ & $1.32 \times 10^{5}$ & 99.78 & 136.48 & 58.84 \\
\hline AlkalineILs & $5.44 \times 10^{9}$ & $1.47 \times 10^{7}$ & $9.17 \times 10^{14}$ & 110.81 & 96.48 & 153.47 \\
\hline
\end{tabular}

Table 3. Pre-exponential factor and activation energy of fructose conversion.

$$
\begin{aligned}
& \text { Arrhenius Equation: } k=A \exp \left(-\frac{E a}{R T}\right) \\
& \text { The deformation: } \ln k=-\frac{E a}{R T}+\ln A
\end{aligned}
$$

Figure $5 \mathrm{~A}, \mathrm{~B}$ show relationship of $\ln k$ and $-10^{-4} / \mathrm{RT}$, using the rate constants, an Arrhenius plot is generated based on Eq. (8) \& (9). The kinetic parameters for ILs catalyzed dehydration of fructose to 5-HMF, $E a$ (slope) and $A$ (intercept is $\ln A$ ) are summarized in Table 3.

The values of activation energy and pre-exponential factor of 5-HMF degradation $\left(A_{2}\right.$ and $E a_{2}, 1.47 \times 10^{7}$ and $\left.96.48 \mathrm{~kJ} \cdot \mathrm{mol}^{-1}\right)$ catalyzed by alkaline ILs are lower than those $\left(4.95 \times 10^{13}\right.$ and $\left.136.48 \mathrm{~kJ} \cdot \mathrm{mol}^{-1}\right)$ in acidic catalyst system, meaning that 5 -HMF is degraded into small molecules such as levulinic acid, formic acid much faster than that catalyzed by [MIMPS $]_{3} \mathrm{PW}_{12} \mathrm{O}_{40}$. Moreover, higher $E a_{1}$ and $E a_{3}\left(110.81 \mathrm{~kJ} \cdot \mathrm{mol}^{-1}\right.$ and $\left.153.47 \mathrm{~kJ} \cdot \mathrm{mol}^{-1}\right)$ cause the reaction rate greatly influenced by temperature, and can thus quickly reach the reaction equilibrium.

\section{Discussion}

An efficient synthesis of 5-HMF by conversion of disaccharide has been achieved using ILs as catalysts. Several factors were found to influence the yield of 5-HMF from cellobiose, such as reaction temperature, the dosage of $\mathrm{IL}$, solvent and reaction time. Of the ILs tested, $[\mathrm{AEMIM}] \mathrm{BF}_{4}$ showed the highest catalytic activity and selectivity, and a $68.21 \%$ yield of $5-\mathrm{HMF}$ from sucrose was obtained after $6 \mathrm{~h}$ when it was employed at $160^{\circ} \mathrm{C}$. At same reaction, $5-\mathrm{HMF}$ yield was $24.73 \%$ in the conversion of cellobiose. In addition, a kinetic analysis was carried out on both acidic and alkaline IL-catalyzed system, suggesting main side reaction in the conversion of fructose catalyzed by acidic and alkaline IL was polymerization of fructose and 5-HMF degradation, respectively. Although the catalyzed mechanism needs to be further studied, the results obtained from the present investigation might encourage researchers to consider ILs as potential catalysts for organic reactions rather than simply as solvents.

\section{Methods}

Materials and experimental methods. N-methylimidazole was purchased from Aladdin Chemical Co., Ltd. 2-Chloroethylaminehydrochloride, dichloroethanol, and acetone from Shanghai Jingchun Chemical Reagent Company. Dimethylsulfoxide (DMSO), dimethyl acetamide (DMAc), N,N-dimethylformamide(DMF), n-butanol, 2-butanol, methylisobutylketone (MIBK), sodium tetrafluoroborate, ether, ethanol, sodium borate $\left(\mathrm{NaBF}_{4}\right)$,sucrose, cellobiose, glucose and fructose were purchased from Beijing Chemical Reagent Company. All reagents were used as supplied without further purification.

The hydroxy-functionalized ILs, [AEMIM] $\mathrm{BF}_{4}{ }^{27-29}$ was synthesized as follows: 0.10 mol N-methylimidazole $(8.20 \mathrm{~g})$ and $0.10 \mathrm{~mol}$ 2-bromoethylamine hydrobromide $(20.50 \mathrm{~g})$ were dissolved into $60 \mathrm{ml}$ ethanol, and stirred at $80^{\circ} \mathrm{C}$ under nitrogen atmosphere. After $24 \mathrm{hrs}$, the mixture was filtered, and washed with ethanol for three times. The residue was dissolved into $40 \mathrm{ml}$ of $\mathrm{CH}_{3} \mathrm{CN} / \mathrm{H}_{2} \mathrm{O}(1: 1, \mathrm{v} / \mathrm{v})$ with the addition of $0.10 \mathrm{~mol} \mathrm{NaBF}_{4}(10.98 \mathrm{~g})$. It was stirred at $30^{\circ} \mathrm{C}$ for another $24 \mathrm{hrs}$ and then, evaporated in vacuum. The obtained yellow liquid was washed 


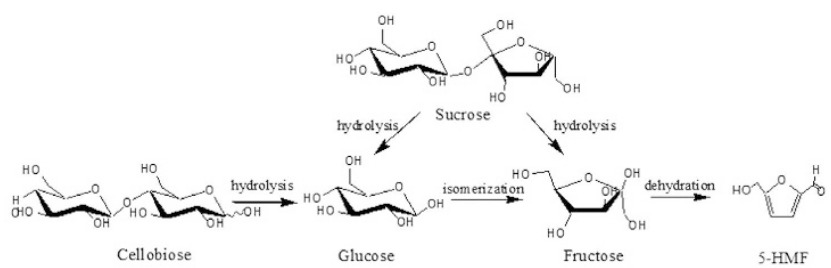

Figure 6. Reaction process for the dehydration.

with chloroform and ether for three times, respectively. After drying at $60^{\circ} \mathrm{C}$ for $24 \mathrm{~h}$ in vacuum, pure [AEMIM] $\mathrm{BF}_{4}$ was obtained with the yield of $74.4 \%$.

Other ILs were also synthesized by two-step methods: synthesis of imidazolium chloride precursor followed by anion exchange as described by Cai et al. ${ }^{13}$. ILs containing different cations and anions, including $[\mathrm{MIMPS}]_{3} \mathrm{PW}_{12} \mathrm{O}_{40}{ }^{36},[\mathrm{BMIM}] \mathrm{OH}^{37},[\mathrm{EMIM}] \mathrm{OH}^{38}$ and $[\mathrm{BMIM}] \mathrm{HCO}_{3}{ }^{39}$ were prepared.

General procedure for the conversion of carbohydrates to 5-HMF. In the dehydration reaction, $0.5 \mathrm{~g}$ of carbohydrate, $0.25 \mathrm{~g}$ of catalyst and $60 \mathrm{~mL}$ of solvent were mixed in a $100-\mathrm{mL}$ flask equipped with a condenser and heated to certain reaction temperature. After dehydration, the mixture was analyzed by HPLC. During the dehydration reaction, disaccharide was hydrolyzed into glucose and fructose, glucose can be isomerized into fructose and the later loses three water molecules to produce HMF in the presence of catalyst (Fig. 6).

After reaction, the mixture was filtered through $0.45 \mu \mathrm{m}$ pore size membrane and the filtrate was transferred into a volumetric flask and diluted with water. 5-HMF and furfural were analyzed by HPLC (Agilent LC1260 infinity, USA) using a C18 column and UV detector at $35^{\circ} \mathrm{C}$ with methanol / water $(40 / 60, \mathrm{v} / \mathrm{v})$ as eluent at a flow rate of $0.6 \mathrm{~mL} / \mathrm{min}$.

Statistical Analysis. Based on data distribution, one-way and two-way analyses of variance (ANOVA) was used to compare different groups. The $\mathrm{P}$ value was calculated, which less than 0.05 was considered as statistically significant with $\mathrm{P}<0.05$.

\section{References}

1. Falter, C. et al. Glucanocellulosic ethanol: the undiscovered biofuel potential in energy crops and marine biomass. Sci Rep-Uk $\mathbf{5}$, doi: Artn 1372210.1038/Srep13722 (2015).

2. Guo, X. C. et al. Conversion of biomass-derived sorbitol to glycols over carbon-materials supported Ru-based catalysts. Sci Rep-Uk 5, doi: Artn 1645110.1038/Srep16451 (2015).

3. Teong, S. P., Yi, G. S. \& Zhang, Y. G. Hydroxymethylfurfural production from bioresources: past, present and future. Green Chem 16, 2015-2026, doi: 10.1039/c3gc42018c (2014).

4. Wang, J. J. et al. Direct conversion of carbohydrates to 5-hydroxymethylfurfural using Sn-Mont catalyst. Green Chem 14, 2506-2512, doi: $10.1039 / \mathrm{c} 2 \mathrm{gc} 35699 \mathrm{f}(2012)$.

5. Wu, L. Q. et al. Very efficient conversion of glucose to 5-hydroxymethylfurfural in DBU-based ionic Liquids with benzenesulfonate anion. Green Chem 16, 3935-3941, doi: 10.1039/c4gc00311j (2014).

6. Tuteja, J., Choudhary, H., Nishimura, S. \& Ebitani, K. Direct Synthesis of 1,6-Hexanediol from HMF over a Heterogeneous Pd/ZrP Catalyst using Formic Acid as Hydrogen Source. Chemsuschem 7, 96-100, doi: 10.1002/cssc.201300832 (2014).

7. Zhang, Z. H., Liu, B., Lv, K. L., Sun, J. \& Deng, K. J. Aerobic oxidation of biomass derived 5-hydroxymethylfurfural into 5-hydroxymethyl-2-furancarboxylic acid catalyzed by a montmorillonite K-10 clay immobilized molybdenum acetylacetonate complex. Green Chem 16, 2762-2770, doi: 10.1039/c4gc00062e (2014).

8. Wang, H. L. et al. Graphene oxide as a facile acid catalyst for the one-pot conversion of carbohydrates into 5-ethoxymethylfurfural. Green Chem 15, 2379-2383, doi: 10.1039/c3gc41109e (2013).

9. Subbiah, S., Simeonov, S. P., Esperanca, J. M. S. S., Rebelo, L. P. N. \& Afonso, C. A. M. Direct transformation of 5-hydroxymethylfurfural to the building blocks 2,5-dihydroxymethylfurfural (DHMF) and 5-hydroxymethyl furanoic acid (HMFA) via Cannizzaro reaction. Green Chem 15, 2849-2853, doi: 10.1039/c3gc40930a (2013).

10. Rass, H. A., Essayem, N. \& Besson, M. Selective aqueous phase oxidation of 5-hydroxymethylfurfural to 2,5-furandicarboxylic acid over Pt/C catalysts: influence of the base and effect of bismuth promotion. Green Chem 15, 2240-2251, doi: 10.1039/c3gc40727f (2013).

11. Yang, Z. Z., Deng, J., Pan, T., Guo, Q. X. \& Fu, Y. A one-pot approach for conversion of fructose to 2,5-diformylfuran by combination of $\mathrm{Fe}_{3} \mathrm{O}_{4}$-SBA-SO $\mathrm{S}_{3} \mathrm{H}$ and K-OMS-2. Green Chem 14, 2986-2989, doi: 10.1039/c2gc35947b (2012).

12. Xie, J. H., Nie, J. F. \& Liu, H. C. Aqueous-phase selective aerobic oxidation of 5-hydroxymethylfurfural on Ru/C in the presence of base. Chinese J Catal 35, 937-944, doi: 10.1016/S1872-2067(14)60136-4 (2014).

13. Cai, Y. Q., Peng, Y. Q. \& Song, G. H. Amino-functionalized ionic liquid as an efficient and recyclable catalyst for Knoevenagel reactions in water. Catal Lett 109, 61-64, doi: 10.1007/s10562-006-0057-3 (2006).

14. Hou, Y. D. \& Wang, X. C. Ionic Liquid Co-catalyzed Artificial Photosynthesis of CO. Sci Rep-Uk 3, doi: Artn 105610.1038/Srep01056 (2013).

15. Parvulescu, V. I. \& Hardacre, C. Catalysis in ionic liquids. Chem Rev 107, 2615-2665, doi: 10.1021/cr050948h (2007).

16. Weber, C. C., Masters, A. F. \& Maschmeyer, T. Structural features of ionic liquids: consequences for material preparation and organic reactivity. Green Chem 15, 2655-2679, doi: 10.1039/c3gc41313f (2013).

17. Zakrzewska, M. E., Bogel-Lukasik, E. \& Bogel-Lukasik, R. Ionic Liquid-Mediated Formation of 5-Hydroxymethylfurfural-A Promising Biomass-Derived Building Block. Chem Rev 111, 397-417, doi: 10.1021/cr100171a (2011).

18. Chun, J. A., Lee, J. W., Yi, Y. B., Hong, S. S. \& Chung, C. H. Catalytic production of hydroxymethylfurfural from sucrose using 1-methyl-3-octylimidazolium chloride ionic liquid. Korean J Chem Eng 27, 930-935, doi: 10.1007/s11814-010-0167-x (2010).

19. Wang, T. F., Nolte, M. W. \& Shanks, B. H. Catalytic dehydration of C-6 carbohydrates for the production of hydroxymethylfurfural (HMF) as a versatile platform chemical. Green Chem 16, 548-572, doi: 10.1039/c3gc41365a (2014).

20. Zhao, H. B., Holladay, J. E., Brown, H. \& Zhang, Z. C. Metal chlorides in ionic liquid solvents convert sugars to 5-hydroxymethylfurfural. Science 316, 1597-1600, doi: 10.1126/science.1141199 (2007). 
21. Ilgen, F. et al. Conversion of carbohydrates into 5-hydroxymethylfurfural in highly concentrated low melting mixtures. Green Chem 11, 1948-1954, doi: 10.1039/b917548m (2009).

22. Lima, S. et al. Conversion of mono/di/polysaccharides into furan compounds using 1-alkyl-3-methylimidazolium ionic liquids. Appl Catal a-Gen 363, 93-99, doi: 10.1016/j.apcata.2009.04.049 (2009).

23. $\mathrm{Hu}$, S. Q. et al. Direct conversion of inulin to 5-hydroxymethylfurfural in biorenewable ionic liquids. Green Chem 11, 873-877, doi: 10.1039/b822328a (2009).

24. Luo, S. Z. et al. Functionalized ionic liquids catalyzed direct aldol reactions. Tetrahedron 63, 1923-1930, doi: 10.1016/j. tet.2006.12.079 (2007).

25. Xu, J. M., Qian, C., Liu, B. K., Wu, Q. \& Lin, X. F. A fast and highly efficient protocol for Michael addition of N-heterocycles to alpha,beta-unsaturated compound using basic ionic liquid [bmIm] OH as catalyst and green solvent. Tetrahedron 63, 986-990, doi: 10.1016/j.tet.2006.11.013 (2007)

26. Yu, Y. H. et al. Ship-in-a-bottle synthesis of amine-functionalized ionic liquids in NaY zeolite for $\mathrm{CO} 2$ capture. Sci Rep-Uk 4, doi: Artn 599710.1038/Srep05997 (2014).

27. Li, W. J. et al. Switching the basicity of ionic liquids by CO(2). Green Chem 10, 1142-1145, doi: 10.1039/b811624e (2008).

28. Sun, H., Zhou, X. Q., Xue, Z. M., Zhou, Z. Y. \& Mu, T. C. Theoretical investigations on the reaction mechanisms of aminefunctionalized ionic liquid [aEMMIM][BF4] and CO2. Int J Greenh Gas Con 20, 43-48, doi: 10.1016/j.ijggc.2013.10.025 (2014),

29. Sharma, P. et al. Solubility of carbon dioxide in amine-functionalized ionic liquids: Role of the anions. Chem Eng J 193, 267-275, doi: 10.1016/j.cej.2012.04.015 (2012).

30. Qu, Y. S., Huang, C. P., Zhang, J. \& Chen, B. H. Efficient dehydration of fructose to 5-hydroxymethylfurfural catalyzed by a recyclable sulfonated organic heteropolyacid salt. Bioresource Technol 106, 170-172, doi: 10.1016/j.biortech.2011.11.069 (2012).

31. Qi, X. H., Watanabe, M., Aida, T. M. \& Smith, R. L. Catalytic conversion of cellulose into 5-hydroxymethylfurfural in high yields via a two-step process. Cellulose 18, 1327-1333, doi: 10.1007/s10570-011-9568-1 (2011).

32. Tian, Y. K., Deng, J., Pan, T., Guo, Q. X. \& Fu, Y. Dehydration of Glucose and Fructose into 5-Hydroxymethylfurfural Catalyzed by Lewis Acid in Ionic Liquids. Chinese J Catal 32, 997-1002, doi: 10.3724/Sp.J.1088.2011.01258 (2011).

33. Lentini, S., Galloni, P., Garcia-Bosch, I., Costas, M. \& Conte, V. Ionic liquids as reaction media in catalytic oxidations with manganese and iron pyridyl triazacyclononane complexes. Inorg Chim Acta 410, 60-64, doi: 10.1016/j.ica.2013.10.016 (2014).

34. Waseem, M. A. et al. [bmIm] OH catalyzed hetero-cyclisation of o-halobenzoic acid and alkyne: A green approach to synthesize isocoumarins. Catal Commun 55, 70-73, doi: 10.1016/j.catcom.2014.06.010 (2014).

35. Qin, W. D., Wei, H. P. \& Li, S. F. Y. 1,3-dialkylimidazolium-based room-temperature ionic liquids as background electrolyte and coating material in aqueous capillary electrophoresis. J Chromatogr A 985, 447-454, doi: 10.1007/s10570-011-9568-1 (2003).

36. Ma, Y. Q. \& Wang, R. H2S Absorption Capacity of Ionic Liquid-MDEA-H2O Combined Desulfurizers. Chem J Chinese U 35, 1515-1522, doi: 10.7503/cjcu20140108 (2014).

37. Qu, Y. S., Song, Y. L., Huang, C. P., Zhang, J. \& Chen, B. H. Alkaline Ionic Liquids as Catalysts: A Novel and Green Process for the Dehydration of Carbohydrates To Give 5-Hydroxymethylfurfural. Ind Eng Chem Res 51, 13008-13013, doi: 10.1021/ie300140g (2012).

38. Qi, X. H., Watanabe, M., Aida, T. M. \& Smith, R. L. Efficient process for conversion of fructose to 5-hydroxymethylfurfural with ionic liquids. Green Chem 11, 1327-1331, doi: 10.1039/b905975j (2009).

39. Eminov, S., Wilton-Ely, J. D. E. T. \& Hallett, J. P. Highly Selective and Near-Quantitative Conversion of Fructose to 5-Hydroxymethylfurfural Using Mildly Acidic Ionic Liquids. Acs Sustain Chem Eng 2, 978-981, doi: 10.1021/sc400553q (2014).

\section{Acknowledgements}

The work was financially supported by the National Natural Science Foundation of China (No. 21276259), International (Regional) Cooperation And Exchange Project (No. 21450110062), National High Technology Research and Development Program of China (863 Program: 2012AA022301), University-Institute Cooperation Funds from University of the Chinese Academy of Sciences (No. Y552017Y00) and Technology Plan Projects of Beijing (No. Z14110000061404).

\section{Author Contributions}

Y.Q., C.H. and J.X. conceived and designed experiments. Y.Q., L.L., Q.W. and P.O.P. carried out experiments. Y.Q., L.L., Q.W., P.O.P. and J.X. analyzed data and prepared figures. Y.Q., Q.W., P.O.P. and J.X. wrote the manuscript. All authors reviewed the manuscript.

\section{Additional Information}

Competing financial interests: The authors declare no competing financial interests.

How to cite this article: Yongshui, Q. et al. One-pot conversion of disaccharide into 5-hydroxymethylfurfural catalyzed by imidazole ionic liquid. Sci. Rep. 6, 26067; doi: 10.1038/srep26067 (2016).

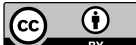

This work is licensed under a Creative Commons Attribution 4.0 International License. The images or other third party material in this article are included in the article's Creative Commons license, unless indicated otherwise in the credit line; if the material is not included under the Creative Commons license, users will need to obtain permission from the license holder to reproduce the material. To view a copy of this license, visit http://creativecommons.org/licenses/by/4.0/ 